\title{
An estimation of the diffusion coefficient of galactic cosmic rays in the heliosphere near the Earth
}

\author{
H.Kojima ${ }^{* a}$, S.Shibata $^{b}$, A.Oshima $^{b}$, Y.Hayashi ${ }^{c}$, S.Kawakami $^{c}$, I.Morishita $^{d}$, \\ T.Nakamura ${ }^{e}$, T.Nonaka ${ }^{f}$, S.Ogio ${ }^{c}$, H.Takamaru ${ }^{b}$, K.Tanaka $^{g}$, N.Ito ${ }^{c}$, T.Matsuyama ${ }^{c}$, \\ K.Yamazaki ${ }^{c}$, M.Tokumaru ${ }^{h}$, S.K.Gupta ${ }^{i}$, H.M.Antia ${ }^{i}$, S.R.Dugad ${ }^{i}$, P.K.Mohanty $^{i}$, \\ A.Jain ${ }^{i}$, B.S.Rao ${ }^{i}$ \\ ${ }^{a}$ Faculty of Engineering, Aichi Institute of Technology, Toyota, Aichi 470-0392, Japan \\ ${ }^{b}$ Faculty of Engineering, Chubu University, Kasugai, Aichi 487-8501, Japan \\ ${ }^{c}$ Graduate School of Science, Osaka City University, Osaka 558-8585, Japan \\ ${ }^{d}$ School of Health Sciences, Asahi University, Mizuho, Gifu 501-0296, Japan \\ ${ }^{e}$ Faculty of Science, Kochi University, Kochi 780-8520, Japan \\ ${ }^{f}$ Institute for Cosmic Ray Research, University of Tokyo, Kashiwa, Chiba 277-8582, Japan \\ ${ }^{g}$ Graduate School of Information Sciences, Hiroshima City University, Hiroshima 731-3194, \\ Japan \\ ${ }^{h}$ Solar-Terrestrial Environment Laboratory, Nagoya University Furo-cho, Aichi, Japan \\ ${ }^{i}$ Tata Institute of Fundamental Research, Mumbai, India \\ E-mail: hkojima@aitech.ac.jp
}

\begin{abstract}
From the density gradient of galactic cosmic rays derived from the Swinson flow and the regression coefficients between the intensity variations of cosmic rays and the solar wind velocity, we have derived the diffusion coefficient and the scattering mean free path of galactic cosmic rays in the heliosphere near the Earth. In this analysis we have used the data obtained by the large area muon tracking detectors of GRAPES-3 and assumed the simplified diffusion-convection equation of cosmic rays in the heliosphere. We will explain the method we have adopted in the estimation of the diffusion coefficient and the scattering mean free path of galactic cosmic rays in this paper.
\end{abstract}

The 34th International Cosmic Ray Conference,

30 July- 6 August, 2015

The Hague, The Netherlands

\footnotetext{
* Speaker.
} 


\section{Introduction}

So called solar-time modulation of cosmic rays, which is observed as an anisotropy of cosmic ray intensity variation in the heliosphere, has been investigated by E. N. Parker[1] and summarised into diffusion-convection theory. This theory has two specific parameters, namely the diffusion coefficient and the solar wind velocity. The diffusion coefficient in the theory, which is an expression of diffusive flow of cosmic ray particles in the heliosphere, plays an equivalent role to a mean free path of cosmic rays in the heliosphere. If one of two parameters was determined, the other one can be automatically calculated theoretically.

The diffusion-convection theory by E. N. Parker initially appeared to explain the propagation of cosmic rays in the heliosphere, however it was later applied to an explanation of the shock acceleration mechanism of cosmic rays near the super novae explosions by Axford et al.[2]. These historical fact implies that if the diffusion coefficient in the diffusion-convection theory were obtained by the ground based experiment of galactic cosmic rays, it could have a capability of explaining the mechanism of cosmic rays propagation in the universe.

To determine the parameters in the diffusion-convection theory, there have been many challenges done by many solar and cosmic ray physicists by means of artificial satellites that measures the Interplanetary Magnetic Field (IMF) and solar wind velocity and low energy charged particles and so on, and computer simulations and ground based cosmic ray experiment such as neutron measurement. In this paper, we adopted a new method to determine these parameters focusing on even higher energy cosmic rays of $\geq 10 \mathrm{GeV}$, using only IMF data and the data of ground based muon detectors not using any other computer simulations. The methods we adopted in this paper comprises of two different independent methods. The first one is based on the radial density gradient of galactic cosmic rays derived from the Swinson flow which is the streaming perpendicular to the ecliptic plane in the north-south direction observed as a sidereal diurnal variation by the ground level experiments. The second one is based on the regression coefficient which was derived from the relationship between the cosmic ray intensity variation and the variation of the solar wind velocity.

\section{Swinson flow}

The solar wind is responsible for sweeping out the galactic cosmic rays, creating a radial density gradient within the heliosphere. This gradient coupled with the IMF induces a flow of charged particles perpendicular to the ecliptic plane which was measured and correctly explained by Swinson, called 'Swinson flow' [5]. The Swinson flow is observed as a small anisotropy in sidereal time in the cosmic ray flux. Its amplitude and phase were measured using the GRAPES-3 tracking muon telescope data. The Swinson flow results from the streaming of cosmic rays $\mathbf{B} \times \mathbf{G}_{r}$ perpendicular to the ecliptic plane along the north-south direction, here $\mathbf{B}$ is the IMF and $\mathbf{G}_{r}$ the radial density gradient of the cosmic rays in the heliosphere. This streaming results in a diurnal anisotropy in the cosmic ray intensity variation in sidereal time, due to the tilt of the rotation axis of Earth relative to the plane of the ecliptic. Therefore, a maximum counting rate is observed by a muon telescope at $18 \mathrm{~h}$ local sidereal time, if the streaming is downward (north-south) and at $6 \mathrm{~h}$ local sidereal time 
if the streaming is upward (south-north) as shown in Figure1.

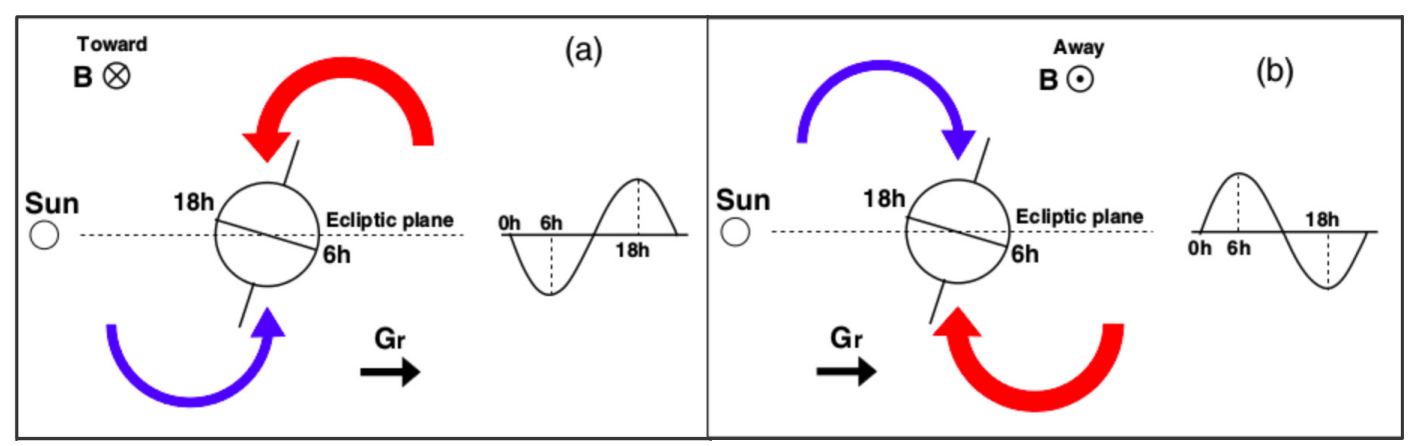

Figure 1: Schematic image of Swinson flow at Earth with tilted rotation axis. Colored arrows indicate flow of cosmic rays in IMF. Curved thick red arrow indicates excess flow and blue thin arrow deficit flow. IMF relative to Sun in, (a) TW sector, sinusoidal flow with maximum at $18 \mathrm{~h}$, (b) Away sector, sinusoidal flow with maximum at $6 \mathrm{~h}$. (TW-AW)/2 removed common systematic effects and yielded actual TW flow.

The variation due to the Swinson flow represented by the (TW-AW)/2 are shown on Figure.2, which displays a minimum at $6 \mathrm{~h}$ sidereal time. The radial density gradient of the cosmic rays results in radially inward diffusion of cosmic rays.

From the averaged IMF of 4.7 nT during the six year interval 2000-2005, and the median rigidity of the primary protons responsible for producing the muons detected by GRAPES- 3 experiment was $77 \mathrm{GV}$, the cosmic ray radial density gradient was estimated to be $0.65 \%$ a.u. ${ }^{-1}$ [3].

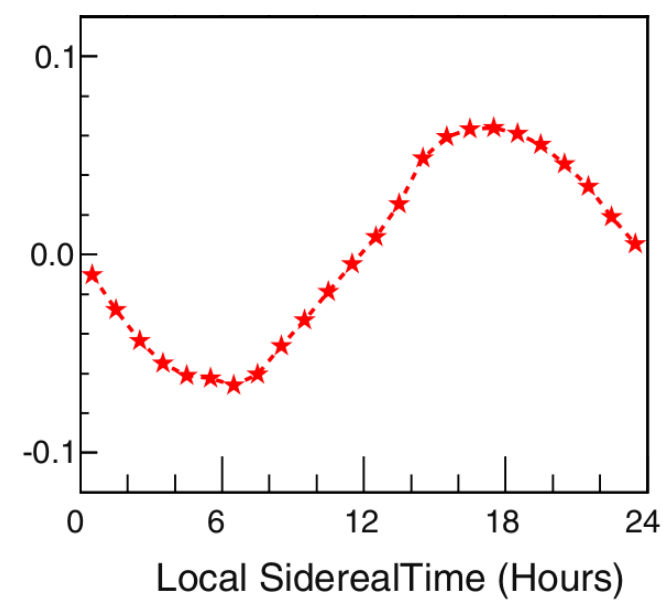

Figure 2: Muon rate variation (\%) due to sidereal diurnal anisotropy for 2000-2005 (TW-AW)/2. The IMF orientation either toward (labeled 'TW') or away ('AW') from the Sun. 


\section{Correlation between cosmic ray intensity variation and solar wind velocity}

The Sun produces a continuous stream of charged particles called solar wind that carries the magnetic field into the heliosphere. The heliosphere comprises an interplanetary region where outward flow of solar wind impedes inflow of galactic cosmic rays and also contains an interplanetary magnetic field (IMF) connected to the Sun. Outflow of solar wind coupled with solar rotation forces the IMF into a spiral form and convects charged particles away from the Sun. Irregularities in the IMF influence convection of cosmic rays through interaction with the solar wind. Any phenomenon occurring in interplanetary space that influences the flux of galactic cosmic rays would also affect intensity of neutrons and muons on the ground. The radial density gradient of cosmic rays reflects the mean value of $V_{S W}$. Solar wind is known to display rapid changes in its velocity $V_{S W}$ and a measurement of variation in muon intensity in response to changes in $V_{S W}$ offers a powerful handle to probe the space weather, wherein muons act as a proxy for galactic cosmic rays.

The correlation between $V_{S W}$ and cosmic rays has been long studied, especially during the active phase of the Sun[7],[8],[9],[10]. Those earlier studies of $V_{S W}$ and cosmic ray intensity, focused on contribution of active regions or specific events such as flares and Coronal Mass Ejections(CMEs) that could have easily resulted in correlated changes between $V_{S W}$ and cosmic rays.

We analyzed the GRAPES-3 data to investigate the correlation of cosmic ray intensity with variation of $V_{S W}$ during low solar activity by eliminating data influenced by Forbush decreases (FDs), ground level enhancements (GLEs) and by various periodic phenomena, such as $27 \mathrm{~d}$ solar rotation, annual, 11 yr solar activity, 22 yr solar magnetic cycle etc, Figure.3. The objective being that if the contribution of change in $V_{S W}$ on cosmic ray intensity could be shown under quiescent solar conditions. Figure. 4 displays the variation of muon intensity $\left(\Delta I_{\mu}\right)$ relative to $V_{S W}$. A clear anticorrelation between $\Delta I_{\mu}$ and $V_{S W}$ with a slope of $(-1.33 \pm 0.07) \times 10^{-5} \% /\left(\mathrm{kms}^{-1}\right)$ was obtained for a median rigidity of $66 \mathrm{GV}[4]$.

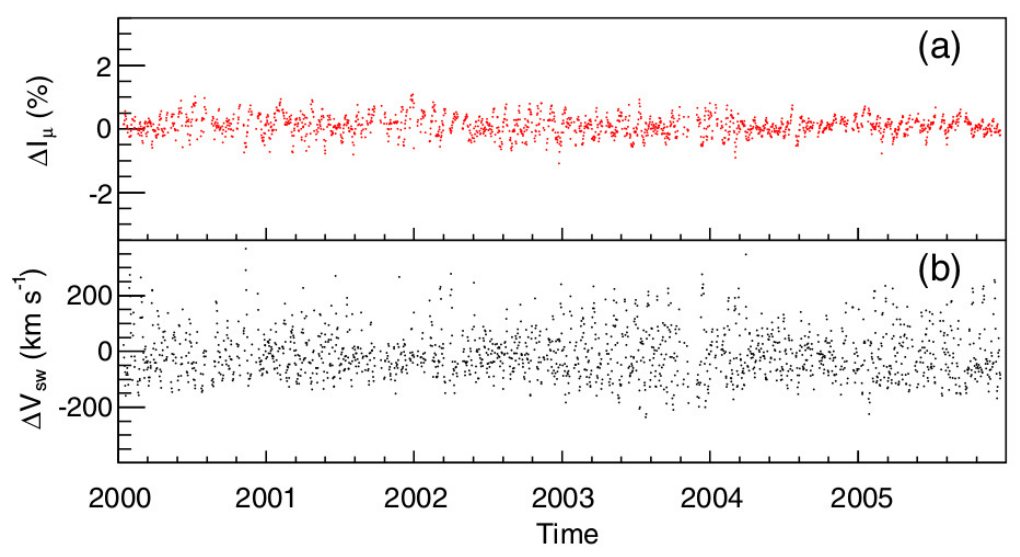

Figure 3: Daily variation after high pass filter applied, (a) muon intensity $\Delta I_{\mu}(\%)$, (b) solar wind velocity $V_{S W}$ relative to respective 6 yr mean. 


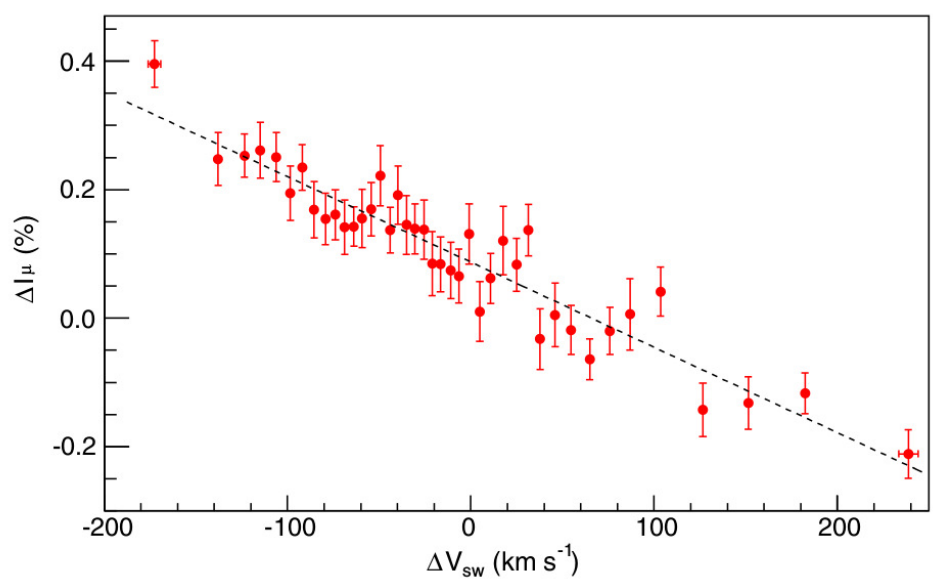

Figure 4: Muon intensity variation $\Delta I_{\mu}(\%)$ correlated with solar wind velocity $V_{S W}$ after binning into 41 intervals.

\section{Method of an estimation of the diffusion coefficient}

According to the abstract of E.N. Parker's paper published in 1965 under the title of "The passage of Energetic Charged Particles through Interplanetary space" (Planet. Space. Sci., 13, $9-49)$, the diffusion coefficient of cosmic rays in order of $10^{17} \sim 10^{18} \mathrm{~m}^{2} / \mathrm{s}$ and a middle value of $3 \times 10^{17} \mathrm{~m}^{2} / \mathrm{s}$ suggests a cosmic ray density gradient of about $10 \% /$ a.u. across the orbit of the Earth.

From the simple one-dimensional diffusion-convection theory, at the steady state of balancing diffusion term and convection one, the cosmic ray density $n(r)$ at the radius $r$ from the center of the Sun is expressed by the differential equation using the velocity of solar wind $v$ and the diffusion coefficient $\kappa$ as follows;

$$
-\kappa \frac{\mathrm{d} n}{\mathrm{~d} r}+v n=0
$$

and the solution of this equation expressed as;

$$
n(r)=n\left(r_{0}\right) \exp \left\{-v\left(r_{0}-r\right) / \kappa\right\}
$$

where $r_{0}$ is the radius of the region of several a.u.. Then we can derive the cosmic ray density gradient as;

$$
\frac{1}{n} \frac{\mathrm{d} n}{\mathrm{~d} r}=\frac{v}{\kappa}
$$

Using this equation, if we adopt the values of $\Delta n / n=10 \%=0.1, \Delta r=1$ a.u. $=1.5 \times 10^{11} \mathrm{~m}$ and $\kappa=3 \times 10^{17} \mathrm{~m}^{2} / \mathrm{s}$, we get the value of solar wind velocity as;

$$
v=\frac{\Delta n}{n} \times \frac{\kappa}{\Delta r}=200 \mathrm{~km} / \mathrm{s}
$$


And, if we adopt the value of $\Delta n / n=0.65 \%=0.0065$, which obtained by H.Kojima et al. and the value of solar wind velocity $v=4.5 \times 10^{5} \mathrm{~m} / \mathrm{s}$ with the same value of $\Delta r$, we get the value of diffusion coefficient as;

$$
\kappa=v \times \Delta r \times\left(\frac{\Delta n}{n}\right)^{-1}=1.0 \times 10^{19} \mathrm{~m}^{2} / \mathrm{s} .
$$

If we assume the cosmic ray density as a function of the velocity of solar wind, the rate of variation of cosmic ray density respond to the variation of solar wind velocity can be derived as;

$$
-\frac{1}{n} \frac{\mathrm{d} n}{\mathrm{~d} v}=\frac{r_{0}-r}{\kappa}
$$

In order to estimate the regression coefficient between the variation of cosmic ray density and the variation of solar wind velocity, if we put the values of $r_{0}-r=1$ a.u. $=1.5 \times 10^{11} \mathrm{~m}$ and $\kappa=3 \times 10^{17} \mathrm{~m}^{2} / \mathrm{s}$ into this equation, we get the result as;

$$
\frac{1}{n} \frac{\Delta n}{\Delta v}=-\frac{r_{0}-r}{\kappa}=-5 \times 10^{-2} \% /(\mathrm{km} / \mathrm{s}) .
$$

Or, if we use the regression coefficient between the variation of cosmic ray density and the variation of solar wind velocity, $-0.00133 \% /(\mathrm{km} / \mathrm{s})$ obtained by H.Kojima et al., we get the value of diffusion coefficient as;

$$
\kappa=-\left(r_{0}-r\right) \times\left(\frac{1}{n} \frac{\Delta n}{\Delta v}\right)^{-1}=1.1 \times 10^{19} \mathrm{~m}^{2} / \mathrm{s}
$$

If we assume the relationship between the mean free pass $\lambda$ and the diffusion coefficient $\kappa$ as;

$$
\kappa=\frac{\lambda c}{3}
$$

where $c$ is the light velocity, we obtain the mean free path as;

$$
\lambda=\frac{3 \kappa}{c}=1.0 \sim 1.1 \times 10^{11} \mathrm{~m} \sim 0.7 \text { a.u. }
$$

\section{Summary}

We performed an estimation of the diffusion coefficient $\kappa$ and the scattering mean free path $\lambda$ of galactic cosmic rays at earth's orbit experimentally with the help of a simple one dimensional diffusion-convection theory by two different independent methods. The first method is based on the radial density gradient $\mathbf{G}_{r}$ of galactic cosmic rays derived from the Swinson flow and the second method is based on the correlation $\Delta I / \Delta V_{S W}$ between the cosmic ray intensity variation and the solar wind velocity. The resultant diffusion coefficients obtained by the two methods, $\kappa=1.0 \times$ $10^{19} \mathrm{~m}^{2} / \mathrm{s}$ and $\kappa=1.1 \times 10^{19} \mathrm{~m}^{2} / \mathrm{s}$, are consistent with each other very well. Both the values $\mathbf{G}_{r}$ and $\Delta I / \Delta V_{S W}$ we have used in this paper were obtained by the large area muon tracking detectors of GRAPES-3 experiment and the solar wind velocity was observed by the OMNI satellite. Though we assumed the very simplified diffusion-convection equation of cosmic rays in the heliosphere, we can emphasize that our procedure for an estimation of diffusion coefficient of galactic cosmic rays experimentally is unique ever done before. 


\section{Acknowledgment}

We thank all the staff in India for their help in the operation, maintenance of the proportional counters and the associated electronics for the GRAPES-3 tracking muon telescope. This work was partially supported by the grants of the Solar Terrestrial Environment Laboratory of Nagoya University, the Institute for Cosmic Ray Research of University of Tokyo, Chubu University, and the National Astronomical Observatory of Japan.

\section{References}

[1] E.N. Parker, Phys. Rev., 110, (1958) 1445.

[2] W.I. Axford, Ap. J. Suppl., 90, (1994) 937.

[3] H. Hkojima, Astroparticle. Physics. , 62, (2015) 21-29.

[4] H. Hkojima, Physical. Review. D, 91, (2015) 121303.

[5] D.B. Swinson, J. Geophys. Res. 74 (1969) 5591; D.B. Swinson, J. Geophys. Res. 81 (1976) 2075.

[6] D. B. Beard, Rep. Prog. Phys. 30, 409 (1967); E. J. Weber and L. Davis Jr., Astrophys. J. 148, 217 (1967).

[7] N. Iucci, M. Parisi, M. Storini, and G. Villoresi, Nuovo Cimento 2, 421 (1979).

[8] Y. Munakata and K. Nagashima, in Proceedings of the 16th ICRC, Kyoto, 1979 (ICRR, University of Tokyo, Tokyo, 1979), Vol. 4, p. 530.

[9] D. Venkatesan, A. K. Shukla, and S. P. Agrawal, Sol. Phys. 81, 375 (1982).

[10] K. Fujimoto, H. Kojima, and K. Murakami, in Proceedings of the 18th ICRC, Bangalore, 1983 (P. V. Ramana Murthy, Bombay, 1983), Vol. 3, p. 267; in Proceedings of the 19th ICRC, La Jolla, 1985 (NASA, Washington, DC, 1985), Vol. 5, p. 262. 